\title{
Constraints on Interstellar Plasma Turbulence Spectrum from Pulsar Observations at the Ooty Radio Telescope
}

\author{
N. D. Ramesh Bhat \\ Max-Planck-Institut für Radioastronomie, Bonn, Germany \\ Yashwant Gupta, A. Pramesh Rao \\ National Centre for Radio Astrophysics, Pune, India
}

\section{Introduction}

Refractive Interstellar Scintillation (RISS) effects on pulsar signals are powerful techniques for discriminating between different models that have been proposed for the power spectrum of plasma density fluctuations in the Interstellar Medium (ISM; e.g. Rickett 1990). The nature of the spectrum is considered to be a major input for understanding the underlying mechanism of interstellar plasma turbulence. Data from our long-term pulsar scintillation observations using the Ooty Radio Telescope (ORT) at $327 \mathrm{MHz}$ are used to investigate the nature of the spectrum in the Local Interstellar Medium (LISM; region within $\sim 1 \mathrm{kpc}$ of the Sun). Dynamic scintillation spectra were obtained for 18 pulsars in the DM range $3-35 \mathrm{pc} \mathrm{cm}^{-3}$ at $\sim 10-100$ epochs spanning $\sim 100-1000$ days during 1993-1995 (Bhat et al. 1999). From these observations, various scintillation properties and the ISM parameters are estimated with accuracies much better than that which has been possible from most earlier data. The time series of parameters, $v i z$., decorrelation bandwidth $\left(\nu_{d}\right)$, scintillation time scale $\left(\tau_{d}\right)$ and the drift slope of intensity scintillation patterns, and pulsar flux density are used to study various observable effects of Interstellar Scintillation, based on which the spectral form is inferred over the spatial scale range $\sim 10^{7} \mathrm{~m}$ to $\sim 10^{13} \mathrm{~m}$.

\section{Results and Conclusions}

The main results from the present study are:

1. Observations show large-amplitude modulations of the diffractive scintillation observables $\left(\nu_{d}\right.$ and $\left.\tau_{d}\right)$ and flux. The measured depths of modulations are considerably larger than the predictions of models based on a thin-screen scattering geometry and a simple Kolmogorov form of density spectrum.

2. The statistical properties of diffractive and refractive scattering angles are used to obtain precise estimates of the spectral slope $(\beta)$ over the spatial scale range $\sim 10^{7} \mathrm{~m}$ to $\sim 10^{11} \mathrm{~m}$. While for 12 pulsars, $\beta$ is found to be consistent with the Kolmogorov index (at $\pm 2-\sigma$ levels), it is found to be significantly larger for 6 pulsars $(11 / 3<\beta<4)$. 
PSR B0834+06

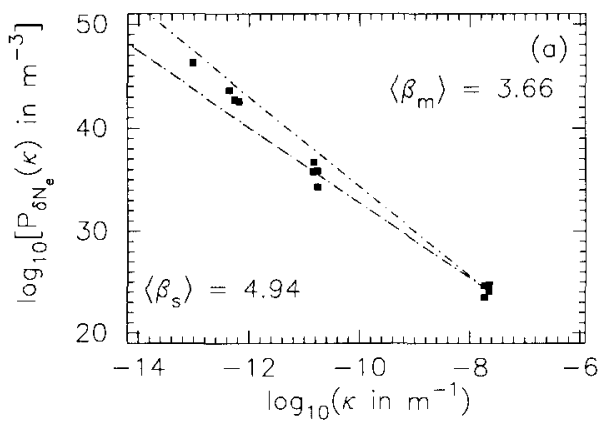

PSR B $1919+21$

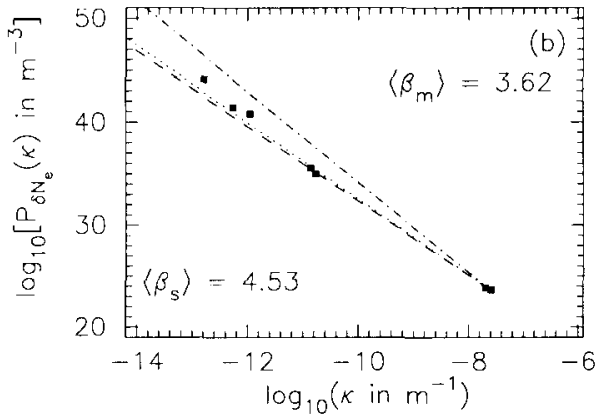

Figure 1. Electron density power spectra for pulsars PSR B0834+06 and PSR B1919+21, determined using various observable effects of interstellar scattering seen in Ooty pulsar observations. The dashed line corresponds to the average slope $\left(\left\langle\beta_{\text {meas }}\right\rangle\right)$ determined by power levels at diffractive and refractive scales. The dotted line represents the Kolmogorov scaling $(\beta=11 / 3)$ and the dot-dashed line $\beta=4$.

3. From the anomalous scintillation behaviour of persistent drift slopes lasting over many months, we infer the power levels at spatial scales $\sim 10^{12}-10^{13}$ $m$ (i.e., 1-2 orders of magnitude larger than refractive scales) to be $2-3$ orders of magnitude larger than that expected from a Kolmogorov form of spectrum (Figure 1).

Although, there are various methods of investigating the nature of the spectrum, measurements based on a particular method give similar implications for the spectral form. A careful consideration of all the available results from the literature and our observations leads to the picture of a Kolmogorov-like spec$\operatorname{tr} 1 \mathrm{~m}(\beta \approx 11 / 3)$ in the spatial scale range $\sim 10^{6} \mathrm{~m}$ to $\sim 10^{11} \mathrm{~m}$, with a low wavenumber enhancement (at $\kappa \sim 10^{-12}-10^{-13} \mathrm{~m}^{-1}$ ). This is not quite in agreement with the results from some of the recent literature, where the spectrum is suggested to be an approximately power-law over the wide range $\sim 10^{8}$ $\mathrm{m}$ to $\sim 10^{13} \mathrm{~m}$ (cf. Armstrong et al. 1995). Further, for several nearby pulsars (distance $\sim 200-700 \mathrm{pc}$ ), the spectrum is found to be somewhat steeper $(11 / 3<\beta<4)$, and there is a weak, systematic trend for a decrease in the spectral slope $(\beta)$ with distance.

A more complete description of this work can be found in Bhat N.D.R., Gupta Y., Rao A.P. 1999, ApJ, 514, 249.

\section{References}

Armstrong J.W., Rickett B.J., Spangler S.R. 1995, ApJ, 443, 209

Bhat N.D.R., Rao A.P., Gupta Y. 1999, ApJS, 121, 483

Rickett B.J. 1990, ARA\&A, 28, 561 